\title{
Contrast Enhancement and Optimization for Underwater Images
}

\author{
B. Zhang \\ Department of Computer of \\ Chongqing Industry Polytechnic \\ College \\ Chongqing, China
}

\author{
W.N. Huang Z.J. Fang X.F. Lv \\ College of Automatics of Chongqing \\ University of Post and Telecomm. \\ Chongqing, China
}

\author{
Z.X. Xie \\ Dept of Biomedical engineering of \\ Chongqing Medical University \\ Chongqing China
}

\begin{abstract}
A new view about underwater imaging was proposed in this paper that water self still is an imaging object, and a new synthetic model about underwater image was also presented, that is, the resulting image is a synthetic image made from the images of underwater object and water self. Seeing that the present situation which the visual quality of the resulting image made by current restoration methods about underwater image is not satisfied, a new restoration method about underwater image, that is a systematic method called removing water-compensating attenuation-optimization, was proposed,. The results demonstrated that here proposed method is better than some of other methods.
\end{abstract}

Keywords- Underwater Image; Scattering; Absorption; Beam Hardening; Image Optimization

\section{INTRODUCTION}

The application of underwater imaging is wider and wider, involving a wide field of science and technology, such as deep-sea prospecting and mining, underwater communication, underwater engineering, underwater navigation and control, archaeology, marine biology, diving science and technology, and so on. Now, underwater imaging has become the public plaything from expert tools. In the underwater vision, in addition to the issue that is the two-dimensional map of three-dimensional scenery, the extent that the light passing through water is absorbed and scattered varies with distance and thereby the extent of degradation of an image visibility varies with the distance, thus making the underwater image restoration has become more difficult[1-5].

\section{INTERACTION BETWEEN LIGHT AND WATER}

\section{A. Attenuation law}

The attenuation law of object to light is the classical optics problem, known as Beer-Lambert law as follows.

$$
-d I=\beta I d l=\alpha c I d l
$$

Integrating formula (1) can be get,

$$
I=I_{0} e^{-\beta \cdot l}=I_{0} e^{-\alpha c l}
$$

where $l$ represents the length of light propagation path, and it can also be expressed as a distance from the imaged surface to the imaging device. In the condition of underwater imaging, it expresses the distance from the imaged surface to imaging device. $\boldsymbol{I}_{\boldsymbol{0}}$ and $\boldsymbol{I}$ express the incident light intensity and emergent light intensity, respectively. In the condition of underwater imaging, the former expresses the reflection light intensity from the imaged object surface of underwater, and the latter expresses the light intensity received by the imaging device.

\section{B. Water is also an imaging object}

The view that water is an imaging object does not appear in existing literature, but it is a fact! If the water could not be imaged, how people can see the water? Continuous medium between the imaging object and imaging device absorb both the light intensity, $\boldsymbol{I}_{\boldsymbol{0}}$, of intrinsic image of underwater object and the water itself has to form its image, called the intrinsic image of water. The resulting image formed on the imaging device is the synthesis of intrinsic image absorbed by water and that of water itself. The ultimate effects are similar to image overlapping effect of traditional X-ray camera in medical. On two synthesis model, there is additive model [3], product model[1], convolution model[2,3], weighted sums model[4] and another weighted sums model that we will introduced here.

Assuming that at any point $(\mathrm{x}, \mathrm{y})$ in the underwater images, the chroma of water image is $\boldsymbol{L}(\boldsymbol{x}, \boldsymbol{y}, \boldsymbol{C H})$, and the images of scene is $\boldsymbol{S}(\boldsymbol{x}, \boldsymbol{y}, \boldsymbol{C H})$, the synthesis of weighted sums model is $\boldsymbol{F}(\boldsymbol{x}, \boldsymbol{y}, \boldsymbol{C H})$, then

$$
F(\mathrm{CH})=\frac{L^{2}(\mathrm{CH})}{L(\mathrm{CH})+S(\mathrm{CH})}+\frac{S^{2}(\mathrm{CH})}{L(\mathrm{CH})+S(\mathrm{CH})}
$$

The synthesis of intrinsic image of water and object image, computing by means of formula (3), will result in degeneration, therefore more narrow the spectral bandwidth is narrowed, so its information is decreased.
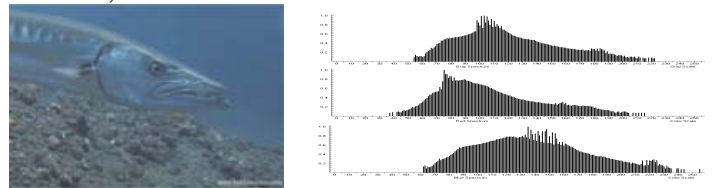

Figure 1 Original underwater image(left)[1] and its color spectra(right) of three color components of red(right top), green(right middle), blue(right bottom)

\section{REMOVING WATER AND ITS REALIZATION}

\section{A. Method of removing water}

Here is an underwater image [1]. The forth order chromatic spectra [6-7] of its RGB components are shown in Fig. 1, and the left (minimum) values and right values are $[37,215]$ for the red, $[59,227]$ for the green, $[62,255]$ for the blue, respectively. Under ideal illumination in clear day, 
there are three spectra with full-bandwidth of [0,255] in three color channels of natural scenery image. We hope so for the restored image.

For the left value, $\boldsymbol{F}_{l}(\boldsymbol{C H})$, and right value, $\boldsymbol{F}_{\boldsymbol{h}}(\boldsymbol{C H})$, of the spectra in Fig. 1, the formula (3) respectively become into the following formulae:

$$
\begin{aligned}
& F_{l}(\mathrm{CH})=\frac{L_{l}^{2}(\mathrm{CH})}{L_{l}(\mathrm{CH})+S_{l}(\mathrm{CH})}+\frac{S_{l}^{2}(\mathrm{CH})}{L_{l}(\mathrm{CH})+S_{l}(\mathrm{CH})} \\
& F_{h}(\mathrm{CH})=\frac{L_{h}^{2}(\mathrm{CH})}{L_{h}(\mathrm{CH})+S_{h}(\mathrm{CH})}+\frac{S_{h}^{2}(\mathrm{CH})}{L_{h}(\mathrm{CH})+S_{h}(\mathrm{CH})}
\end{aligned}
$$

where the subscript $l$ and $h$ respectively denote the left and right values of chromatic spectrum in Fig. 1. Assuming that the original image is standardized (there are full band width for three component spectrum of a standardized image) red $[0,255]$, green $[0,255]$, blue $[0,255]$. If let $F_{l}(\mathrm{CH})=0$, $F_{h}(\mathrm{CH})=255$, it can be know from formulae (8) and (9) that $S_{h}(\mathrm{CH})=255$ and $S_{l}(\mathrm{CH})=0$, and this is ideal underwater image restoration. This means that $L_{h}(\mathrm{CH})=0$ and $L_{l}(\mathrm{CH})=0$, and it can be explained as that the influence of water is eliminated.

\section{B. Realization of removing water algorithm}

In order to realize removing water function, we introduce a transformation called Zadeh-X gray/ chroma transform [8-9], as follows,

$$
T(\mathrm{CH}, x, y)=k \frac{O(C H, x, y)-\text { Theta }}{\text { Delta }}
$$

where $\boldsymbol{C H}=0,1,2$ presents the red, the green and the blue, respectively. $\boldsymbol{k}$ was called an expansion or a contraction factor, here let $\boldsymbol{k}=255$. Theta and Delta were called transformation parameters. $\boldsymbol{O}(\boldsymbol{C H}, \boldsymbol{x}, \boldsymbol{y})$ and $\boldsymbol{T}(\boldsymbol{C H}, \boldsymbol{x}, \boldsymbol{y})$ represents chroma value of a pixel point $(\boldsymbol{x}, \boldsymbol{y})$ before and after transformation at $\boldsymbol{C H}$ channel, respectively. The transformed image is just the original image when Theta $=0$, Delta $=255$. Here, moving water function can be realized when Theta $=F_{h}(\mathrm{CH}), \quad$ Delta $=F_{h}(\mathrm{CH})-F_{l}(\mathrm{CH})$ and to complete transformation of formula (10). For an underwater image taken from [1] and shown in the middle in Fig. 2, the result of transform is shown in the left of Fig. 2 and can be seen that the visual effect is improved obviously.
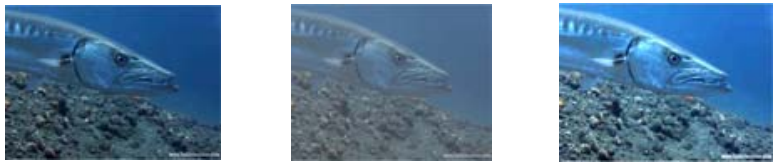

Figure 2 Dewater image(Left) and its Original underwater image(middle), and dewater and $\boldsymbol{R} \boldsymbol{C} \boldsymbol{O}$ result image(Right)

\section{Water attenuation compensation}

Adopting screen coordinates system(SCS) shown as Fig.3, the attenuation law of light beam radiating from ABGP and projecting to the imaging device is as follows.

$$
I=I_{0} e^{-\beta \cdot(B H-y)}
$$

where $\boldsymbol{I}$ is the light intensity arrived at the imaging device. $\boldsymbol{I}_{\boldsymbol{0}}$ is the light intensity emitting from surface, ABGP.
The light intensity attenuated by water, $\boldsymbol{I}$, is computed as follows.

$$
I^{\prime}=I_{0}-I=I_{0} *\left(1-e^{-\beta *(B H-y)}\right)
$$

If the attenuated quantity can be compensated, the attenuation of water is compensated. In practical use, we adopted bandwidth adaptive method, and an adjustable factor $\boldsymbol{\alpha}$ was added ahead of the attenuation term, e.g. the formula (8) become into the following equation.

$$
I^{\prime}=B W *\left(1-\alpha * e^{-\beta *(B H-y)}\right)
$$

where $\boldsymbol{B} \boldsymbol{W}=$ right-left. In the method, attenuation compensation and removing water were completed at the same time. On the basis of subject vision (roughly) and a comprehensive image quality assessment function (finely) $\boldsymbol{C A} \boldsymbol{F}$, the value of $\boldsymbol{\beta}$ was determined experimentally. The concrete data of $\boldsymbol{R C O}$ (removing water-attenuation compensation-optimization, see below) method in table 1 were gotten under the conditions that $\beta=0.006, \alpha=0.006$. This is a set of the compromise constants. In order to obtain the optimal values under certain condition, fine adjustment is needed under the conditions.

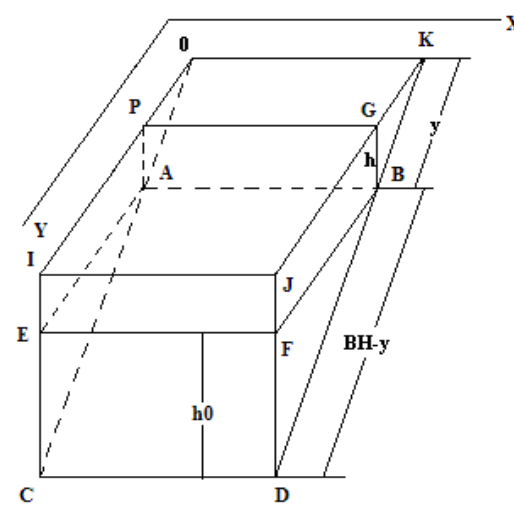

Figure 3 Screen coordinates system

TABLE I. VISIBLE PARAMETERS OF UNDERWATER IMAGE AND THEIR DEWATERED, COMPENSATED AND OPTIMIZED IMAGES

\begin{tabular}{|c|c|c|c|c|c|c|c|c|}
\hline Name & AEI & ACL & ALL & BWF & HF & CAF & ALO & Trans. \\
\hline Left in Fig. 2 & 5.9461 & 1.5584 & 6.4433 & 1.0000 & 0.5938 & 6.4433 & 6.7424 & $62 Z 153[8]$ \\
\hline Middle in Fig.2 & 5.9539 & 0.8241 & 6.8148 & 0.8555 & 0.8771 & 3.3616 & 6.7424 & 0Z255[8] \\
\hline $\begin{array}{c}\text { Right in Fig.2 } \\
\text { Left in Fig 4 d) }\end{array}$ & 6.4251 & 2.0903 & 6.9858 & 1.0000 & 0.6406 & 11.9366 & 6.7424 & RCO \\
\hline Left in Fig 4 a) & 6.9738 & 3.4051 & 6.9915 & 1.0000 & 0.9089 & 23.1399 & 6.6335 & RCO \\
\hline Left in Fig 4 b) & 7.0728 & 2.9476 & 6.7004 & 0.9765 & 0.8477 & 15.9347 & 6.6335 & 0Z255[8] \\
\hline Left in Fig 4 c) & 7.1539 & 3.2745 & 6.5341 & 1.0000 & 0.9805 & 16.9430 & 6.6335 & DCh[4] \\
\hline Left in Fig 4 e) & 7.0605 & 1.6540 & 6.7649 & 1.0000 & 0.9361 & 9.7978 & 6.7424 & WCID[1] \\
\hline Left in Fig 4 f) & 6.6731 & 1.6827 & 6.1240 & 1.0000 & 0.9987 & 6.1402 & 6.7424 & DCh[4] \\
\hline Left in Fig 4 g) & 6.5911 & 1.3567 & 6.7077 & 0.9922 & 0.8490 & 6.9824 & 6.7424 & ChBD[10] \\
\hline Left in Fig 4 h) & 6.3101 & 1.2264 & 6.3464 & 1.0000 & 0.8424 & 4.7318 & 6.7424 & HE[11] \\
\hline Left in Fig. 5 & 6.2430 & 2.1268 & 6.9900 & 1.0000 & 0.5052 & 11.1499 & 6.9637 & OPT \\
\hline Left in Fig 4 i) & 7.1403 & 2.1012 & 6.9608 & 1.0000 & 0.8776 & 14.1938 & 6.9958 & RCO \\
\hline Left in Fig 4 j) & 7.2918 & 1.6254 & 7.0130 & 0.9180 & 0.8555 & 10.3272 & 6.9958 & 0Z255[8] \\
\hline Left in Fig 4 k) & 7.5839 & 2.1159 & 6.6149 & 1.0000 & 0.9974 & 12.3277 & 6.9958 & WCID[1] \\
\hline Left in Fig 4 m) & 7.2847 & 2.1235 & 6.1404 & 1.0000 & 1.0000 & 8.5585 & 6.9958 & DCh[4] \\
\hline
\end{tabular}




\begin{tabular}{|c|c|c|c|c|c|c|c|c|}
\hline Left in Fig 4 n) & 7.4558 & 1.8201 & 6.9462 & 1.0000 & 0.9518 & 12.9638 & 6.9958 & ChBD[10] \\
\hline Left in Fig 4 p) & 7.5161 & 1.7895 & 6.7155 & 1.0000 & 0.9987 & 11.0823 & 6.9958 & HE[11] \\
\hline Name & AEI & ACL & ALL & BWF & HF & CAF & ALO & Trans. \\
\hline
\end{tabular}

Notes: $\boldsymbol{A I E}$ means average information entropy. $\boldsymbol{A C L}$ means average contrast level. $\boldsymbol{A} \boldsymbol{L} \boldsymbol{L}$ means average luminance level. $\boldsymbol{B} \boldsymbol{W} \boldsymbol{F}$ means bandwidth factor. $\boldsymbol{H F}$ means hierarchy factor. $\boldsymbol{C A F}$ means $\mathbf{C}$ omprehensive image quality $\mathbf{A}$ ssessment Eunction. ALO means average luminance of original image. OPT represents optimization. $\boldsymbol{R C O}$ means removing water-attenuation compensationoptimization. Trans. means transformation or algorithms. WCID means wavelength compensation and image dehazing [1]. DCh means dark-Channel [4]. ChBD means chromatism-based dehazing [10]. HE means histogram equalization [11].

\section{OPTIMIZATION}

\section{A. Image optimization after removing water and attenuation compensation}

The vision quality of a restored image by existing method about underwater image restoration often is not satisfied, such as figure $4 \mathrm{c}$ ), e), f), k), m) and p) with low brightness and narrow chromatic spectrum, and thereby, the full spectral space of 0 255 can not be filled. Hence, about restoration of underwater image, there was still a problem to be considered, that is the optimization problem of the restored image, The images in Fig. 4 were taken from the references [1,4], and measurement results of visual parameters were listed in table 1.

Here, our proposed method was called the systematic method of removing water-attenuation compensationoptimization, for short of $\boldsymbol{R} \boldsymbol{C O}$ method. This method contains optimization method of image after removing water and attenuation compensation. The jmage optimization after removing water and attenuation compensation was realized through completing Zadeh-X transformation of formula (6) when let Delta $=\boldsymbol{f}(\boldsymbol{A L L}) * \boldsymbol{A L L}$. Here let $\boldsymbol{f}(\boldsymbol{A} \boldsymbol{L} \boldsymbol{L})=\mathbf{2}$. The results of $\boldsymbol{R O C}$ were shown in the right in Fig. 2 and Fig. 4 a), d) and i). The measurement methods of image vision parameters and the optimization of image vision quality can be seen in [9].
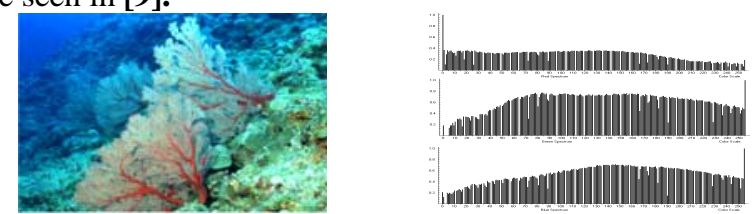

a) Restoring result using our RCO method for underwater image in b) (left) and its chromatic spectra (right)
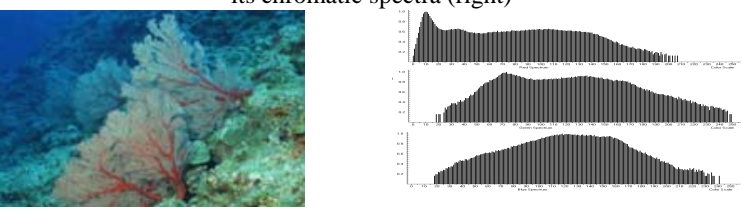

b) Underwater image (left) [4] and its chromatic spectra(right)
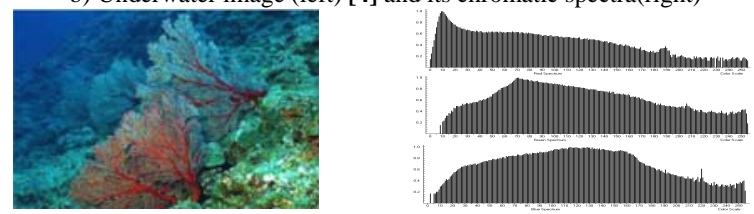

c) Restoring result for image of b) using Chao's method (left) [4] and its chromatic spectra(right)

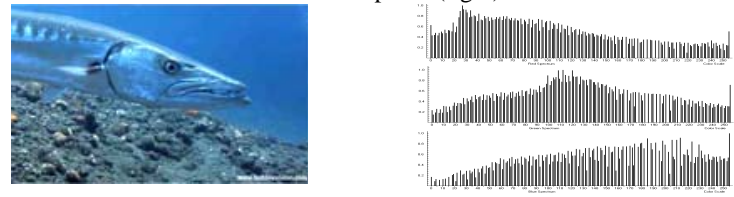

d) Restoring result for image of Fig 1 a) [1] using Our RCO method(left) and its chroma spectra(right)
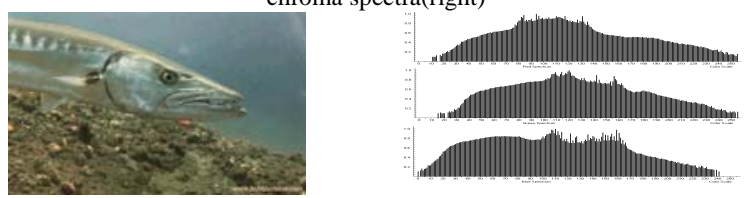

e) Restoring result for image of Fig 1 a) [1] using WCID (left) and its chromatic

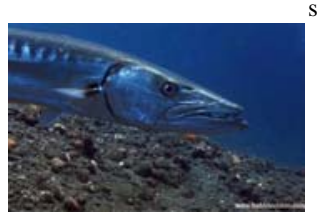
spectra(right)

f) Restoring result for image of Fig 1 a) [1] using DCh [4] (left) and its chromatic spectra(right)
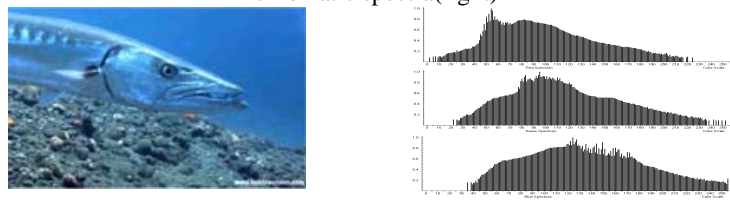

g) Restoring result for image of Fig 1 a) [1] using ChBD [10] (left) and its chromatic spectra(right)
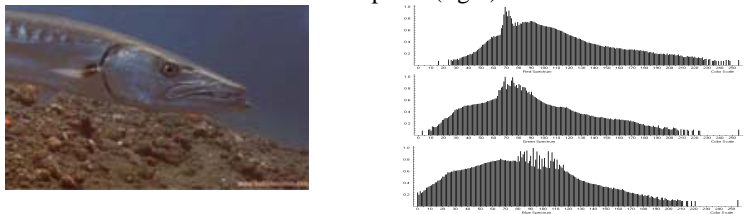

h) Restoring result for image of Fig 1 a) [1] using HE [11] (left) and its chromatic spectra(right)
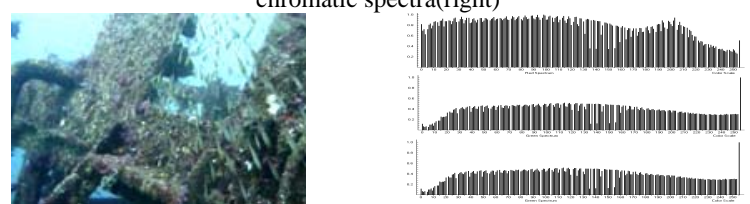

i) Restoring result for image of Fig 4 j) [1] using Our RCO method(left) and its chroma spectra(right)
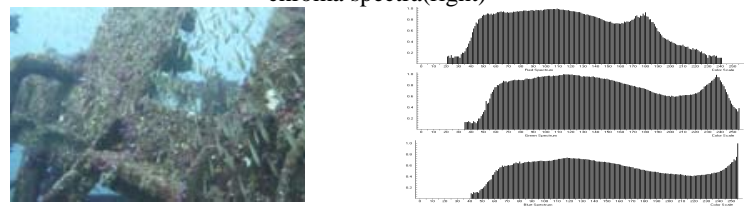

j) Original underwater image(left)[1] and its chromatic spectra(right)
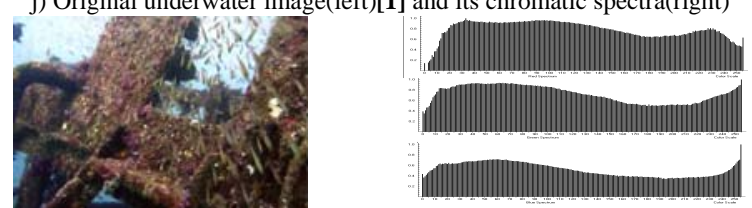

k) Restoring result for image of Fig 4 j) [1] using WCID (left) and its chromatic spectra(right) 

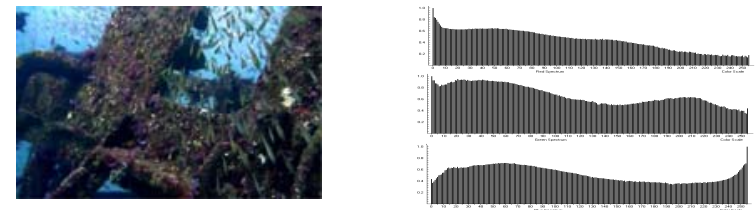

m) Restoring result for image of Fig 4 j) [1] using DCh[4] (left) and its chromatic spectra(right)
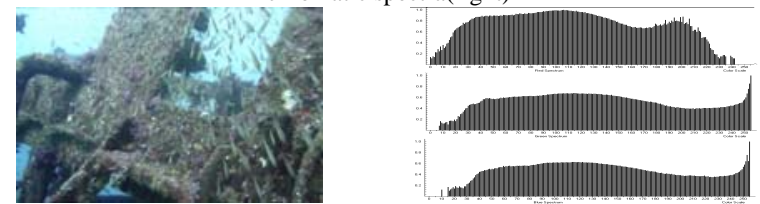

n) Restoring result for image of Fig 4 j) [1] using ChBD[10] (left) and its chromatic spectra(right)
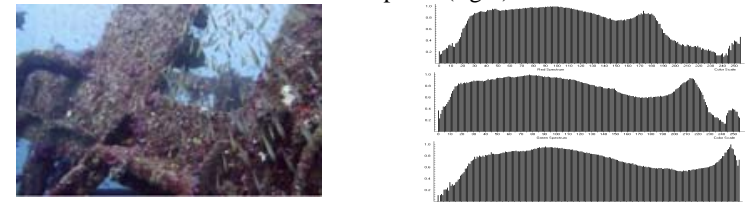

p) Restoring result for image of Fig 4 j) [1] using HE[11] (left) and its chromatic spectra(right)

Figure 4 Comparison among restoring images for underwater images(Left) with various methods and their corresponding chromatic spectra (Right)

\section{SUMMARY OF DATA ANALYSIS RESULTS}

It can be seen from the data of table1 that the common features of restoration results of underwater image for five methods are: (1)The contrast and thereby the image vision quality is increased, seen at the $3^{\text {rd }}$ column in table 1 . (2)The bandwidth of chromatic spectra is increased, seen at the 5th column in table 1. (3)The value of $\boldsymbol{C A F}$ [8] is increased, seen at the 7th column in table 1. That all of $\boldsymbol{C A F}$ values of restored image are higher than that of the original image was demonstrated that the image quality assessment method by means of $\boldsymbol{C A F}$ value based on measuring visual parameters is feasible. (4)Most of the $\boldsymbol{A I E}$ and $\boldsymbol{H F}$ are increased. However, such an increment should be paid attention because there is a suspicion of "creating something out of nothing", so it should be avoided in the general image processing. Increasing of $\boldsymbol{A I E}$ and $\boldsymbol{H F}$ in $\boldsymbol{R C O}$ method could be explained, which results in the nonlinear of removing degeneration and attenuation when removing water.

\section{ABOUT RCO METHOD}

It can be seen from the data in table 1 that all $\boldsymbol{C A F}$ values of the restored images are higher than that of the original images in the five methods in which the underwater images were restored into the clear images. These results illustrate that the five methods all can get the effect enhancing image vision quality. In referred to five methods, the $\boldsymbol{C A F}$ value of $\boldsymbol{R C O}$ method is highest which are 11.9366, 23.1399 and 14.1938 for three different original images. These results can illustrate that the method proposed in this paper is superior to other four methods.

\section{ACKNOWLEDGMENT}

This work is supported by National Natural Science Foundation of China (Grant No.60975008), gratefully.

Corresponding author: Z. X. Xie, Email: bmezxxie@163.com

The first author: Bo Zhang, Email: cqipcmail@163.com

\section{REFERENCES}

[1] J. Y. Chiang and Y. C. Chen. Underwater Image Enhancement by Wavelength Compensation and Dehazing. IEEE Trans. on Image Processing, 21(4): 1756-1769, APRIL 2012.

[2] Y. Y. Schechner and N. Karpel. Clear Underwater Vision. Proceedings of IEEE Conference on Computer Vision and Pattern Recognition, 2004, 1:536-543, 2004.

[3] Y. Wang, B. Wu. Fast Clear Single Underwater Image. 2010 International Conference on Computational Intelligence and Software Engineering (CISE), 2010, 1 - 4 .

[4] L. Chao, W. Meng. Removal of water scattering. 2010 2nd International Conference on Computer Engineering and Technology (ICCET), 2010, V235 - V2-39, 2010.

[5] Y. D. Tian, S. G. Narasimhan. Seeing through water: Image restoration using model-based tracking. 2009 IEEE 12th International Conference, 2009: 2303 - 2310, 2009.

[6] Z. X. Xie, Z.F. Wang, Y. H. Liu, Y.Wang, H. Li. The Theory of Gradually Flattening Gray Spectrum. Chinese Journal of Medical Physics. 23(6):405-407, 426, 2006. (in Chinese)

[7] Z. X. Xie, Z. F. Wang, Y. H. Liu, X. L. Xiong. High Resolution Measurement Method for Gray/Color Information of an Image Used to Lower Level image mining. Chinese Innovation Patent, ZL 200610054324.9, 2008. (in Chinese)

[8]Z.X. XIE, Y. Wang, Z.Y. Peng, Z.F. Wang, Y.H. Liu, H. Li. Image Hiding and Mining Technique Based on the Zadeh-X Transformation. Chinese Journal of Medical Physics. 24(1): 9-11.(in Chinese)

[9] Z.X. Xie, Z.F. Wang, X.F. Lv. Single image quality assessment and creation of the best quality image. Proceedings of 2nd International Conference on Information Science and Engineering, ICISE2010 2010: pp. 4022-4025, 2010

[10] N. Carlevaris-Bianco, A. Mohan, and R. M. Eustice, "Initial results in underwater single image dehazing," in Proc. IEEE OCEANS, 2010, pp. 18, 2010.

[11] K. Iqbal, R. A. Salam, A. Osman, and A. Z. Talib. "Underwater image enhancement using an integrated color model,” Int. J. Comput. Sci., vol. 34 no. 2, pp. 2-12, 2007 